\title{
Long vowels in Mongolian
}

Yumei Sang

ARP, UFR Linguistique, Université Paris Diderot Sorbonne Paris Cité, France

https://doi.org/10.36505/ExLing-2017/08/0025/000327

\begin{abstract}
This paper investigates one of the fundamental and controversial questions in Halh Mongolian: the phonological status of long vowels (VV), especially VV in noninitial syllables and the distribution of VV. This study questions the traditional analysis of vowel quantity based on the single criterion of the realisation of vowel length, which is purely phonetic. This paper takes into consideration the historical development of $\mathrm{VV}$ in Mongolian. It demonstrates that, synchronously, the distinction between long (VV) and short vowels (V) should consider the distinct behaviours that reveal the different nature of these two types of vowels.
\end{abstract}

Key words: Mongolian vowel, long vowel, vowel distribution, vowel devoicing

\section{Introduction}

Mongolian is a language with contrastive vowel quantity. The seven long vowels [a:], [ว:], [ひ:], [e:], [o:], [u:], [i:] contrast with the seven short vowel [a], [०], [च], [e], [o], [u], [i] by a difference in length. However, an important variation of vowel duration is observed both for short and long vowels. This variation of vowel length is the source of different theories with regards to $\mathrm{VV}$ and their distribution. According to a more traditional point of view (Damdinsuren 1959, among others) VV have free distribution and all VV are phonologically long regardless to their position within a word. According to Svantesson et al. (2005), long vowels only appear in the initial position of a word and non-initial VV are treated as short vowels. This later point of view is based on vowel duration.

This present study examines several aspects of acoustic properties of long and short vowels in order to determine the phonological status of non-initial VV sequences and the distribution of long vowels. In addition to the analysis of vowel length and vowel formant structures, a careful study of vowel devoicing is conducted on two types of corpus: target words (controlled speech) and texts (continuous speech). The analysis of vowel devoicing is crucial in understanding the phonemic status of vowel quantity.

Before starting the analysis, the historical development of long vowels in Mongolian should be given briefly. There are two opposite points of view regarding the origin of long vowels. On the one hand long vowels are considered as the result of the merger of two short vowels (Sanzheyev 1953). Roughly, in VCV sequences, the intervocalic consonants ( $\mathrm{y}, \mathrm{g}, \mathrm{Y}, \mathrm{b}$, h) dropped and the two short vowels merged into one long vowel. On the

ExLing 2017: Proceedings of 8th Tutorial and Research Workshop on Experimental Linguistics, 19-22 June, Heraklion, Crete, Greece 
other hand, it is believed that long vowels in Mongolian are primary (Poppe 1962). However, according to these two theories, long vowels appear in all positions within a word and $\mathrm{VV}$ initial and non-initial are developed from the same process.

\section{Methodology}

The data consist of 600 target-words embedded in a frame sentence [pi dedzhelzsen] 'I_ that said, (I said that_) read by six native Mongolian speakers. In total, 1655 long and short vowels are analysed using the signal processing software WinPitch. The segmentation of vowels and consonants is performed manually and visually. The segmentation method is based on oral constriction (Turk et al. 2006). The beginning and the end of F1 and F2 are taken as vowel duration. For vowel devoicing, additional 4 texts containing 1200 words and 2079 long and short vowels are analysed. For the analysis, VV sequences are divided into initial VV and non-initial VV.

\section{Analysis and results}

\section{Measure of vowel length}

The duration of vowels is measured. The average length, standard deviation and median values are given in Table 1. :

Table 1. Average length, standard deviation and median values for initial VV, non-initial VV and V.

\begin{tabular}{cccc}
\hline & Initial $V$ V & Non-initial $V V$ & $V$ \\
\hline Average & 183 & 127 & 75 \\
Stan. deviation & 35 & 33 & 22 \\
Median & 182 & 124 & 75 \\
\hline
\end{tabular}

This table shows that the average lengths are very similar to median values. This indicates the small dispersion of the data. The average means for initial VV, non-initial $\mathrm{VV}$ and $\mathrm{V}$ are $181 \mathrm{~ms}, 127 \mathrm{~ms}$ and $75 \mathrm{~ms}$ respectively. In percentage, short vowels thus represent $42 \%$ of the duration of long vowels and non-initial VV are $69 \%$ of the duration of long vowels. Non-initial VV are exactly in the middle between long and short vowels in terms of duration as shown in Figure 1 below:

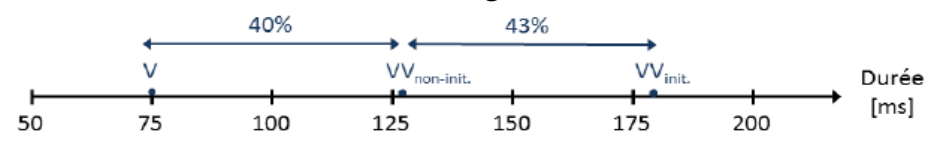

Figure 1. Distance between non-initial VV and V, distance between noninitial VV and V. 
There are thus no reasons to consider that non-initial VV are short rather than long, and vice versa. It also reveals that vowel duration alone cannot constitute a solid factor to determine the phonemic status of vowels, especially for non-initial VV sequences.

\section{Measure of vowel formant structure}

There is evidence from several languages that vowel length impacts vowel quality (Lindblom 1963). Long vowels display more peripheral vowel quality whereas vowels with shorter phonetical length show more centralized vowel triangle. Figure 2 shows the result of formant analysis in Mongolian:

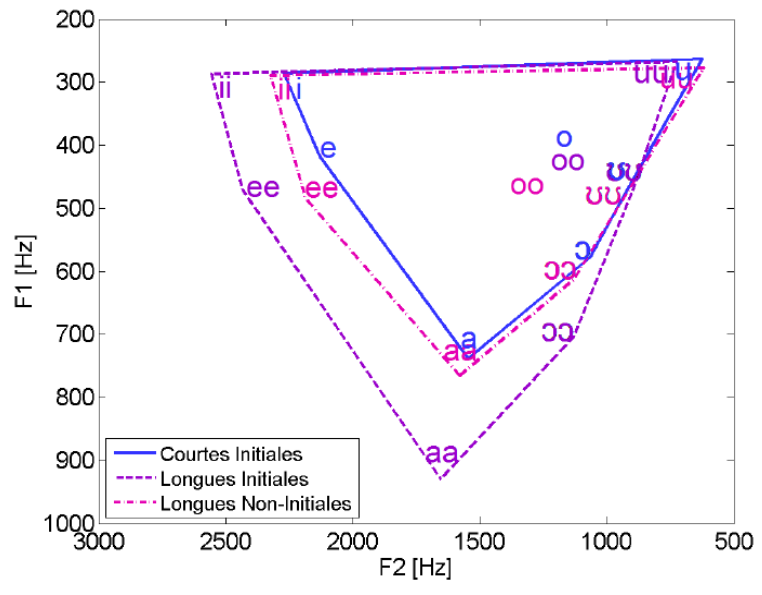

Figure 2. Vowel triangle for initial $\mathrm{VV}$, non-initial $\mathrm{VV}$ and $\mathrm{V}$.

Figure 2 shows that in Mongolian, vowel length does have an impact on vowel quality. Nevertheless, the correlation between vowel quantity and vowel quality seems to depend on each vowel individually rather than on vowel type. The series of $[\mathrm{u}],[\mho]$ and [o] show little difference in vowel quality and some even overlap. This result shows that vowel quality also does not provide a clear indication for the phonological status of non-initial VV.

\section{Vowel devoicing}

The analysis of vowel devoicing here follows the cross linguistic survey of vowel devoicing by Chitoran and Marsico (2010). Vowel devoicing is a process in which vowels are produced with open glottis. Vowel devoicing can be caused by aerodynamic factors (Ohala1983) or/and by the glottal gestural overlap between voiceless consonants and short vowels (Jun and Beckman 1993). The results of the analysis show that $\mathrm{V}$ is highly prone to devoicing, both partially and completely. No case of vowel devoicing is 
observed either for initial and non-initial VV (Sang2016). Figure 3 show one example of vowel devoicing of [i] in [xiffeet] (lesson):

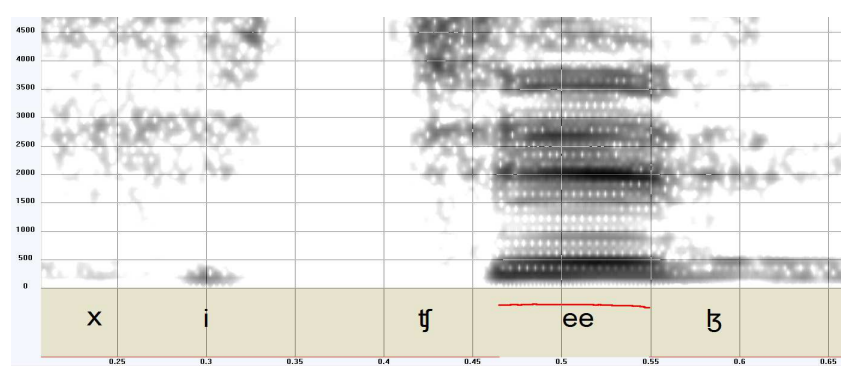

Figure 3. Complete vowel devoicing of [i] and [ee] without devoicing

Initial and non-initial VV behave identically in terms of vowel devoicing and $\mathrm{V}$ differs from them. The identical behaviour of initial and non-initial $\mathrm{VV}$ is governed by their identical underlying status. The longer a vowel, the less it is affected by devoicing.

\section{Conclusion}

This paper provided evidence that vowel length alone cannot be a reliable factor for a distinction between long and short vowels. Other behaviour patterns, such as vowel devoicing must be taken into consideration. It is concluded that initial VV and non-initial VV are both long and therefore long vowels have free distribution in Mongolian.

\section{References}

Chitoran, I., Marsiko, E. Vowel devoicing - an updated phonetic typology. $36^{\text {th }}$ Meeting of Berkeley Society, Berkeley, CA, 2010.

Damdinsuren, St. Orchin tsagiin mongol hel zui. Ulaanbaatar, 1996.

Jun, S.A., and Beckman, M.E., A gestural-overalp analysis of vowel devoicing in Japanese and Korean. 67 ${ }^{\text {th }}$ LSA Annual Meeting. Los Angeles. CA, 1993.

Lindblom, B. Spectrographic study of vowel reduction. JASA, 35(11):1744-1781, 1963.

Ohala, J.J., The origin of sound patterns in vocal tract constraint, in: The production of speech. New York: Springer-Verlag.189-216, P.F.MacNeilage(ed), 1983.

Poppe, N. The primary long vowel in Mongolian. JSFOu, 63(2):1-19, 1962.

Sang, Y. Topics in Mongolian Phonetics and Phonology. Ph.D. thesis. Paris, 2016.

Sanzheyev, G.D., Spavnitelinaya grammatika. Moskva, 1953.

Svantesson, J.-O., Tsendina, A., Karlsson, A., Franzen, V., The phonology of Mongolian. Oxford UniversityPress, 2005.

Turk, A., Nakai, S., Saguhara, M., Acoustic segment duration in prosodic research: A pratical guide. 2006 . 